Research Article

\title{
A New Class of Contact Pseudo Framed Manifolds with Applications
}

\author{
K. L. Duggal \\ Department of Mathematics and Statistics, University of Windsor, Windsor, Ontario N9B3P4, Canada \\ Correspondence should be addressed to K. L. Duggal; yq8@uwindsor.ca
}

Received 26 June 2021; Revised 5 August 2021; Accepted 10 August 2021; Published 27 August 2021

Academic Editor: Luca Vitagliano

Copyright $\odot 2021$ K. L. Duggal. This is an open access article distributed under the Creative Commons Attribution License, which permits unrestricted use, distribution, and reproduction in any medium, provided the original work is properly cited.

In this paper, we introduce a new class of contact pseudo framed (CPF)-manifolds $(M, g, f, \lambda, \xi)$ by a real tensor field $f$ of type $(1,1)$, a real function $\lambda$ such that $f^{3}=\lambda^{2} f$ where $\xi$ is its characteristic vector field. We prove in our main Theorem 2 that $M$ admits a closed 2 -form $\Omega$ if $\lambda$ is constant. In 1976, Blair proved that the vector field $\xi$ of a normal contact manifold is Killing. Contrary to this, we have shown in Theorem 2 that, in general, $\xi$ of a normal CPF-manifold is non-Killing. We also have established a link of CPF-hypersurfaces with curvature, affine, conformal collineations symmetries, and almost Ricci soliton manifolds, supported by three applications. Contrary to the odd-dimensional contact manifolds, we construct several examples of even- and odd-dimensional semi-Riemannian and lightlike CPF-manifolds and propose two problems for further consideration.

\section{Introduction}

Let $\bar{M}$ be an $m$-dimensional real differential manifold. Recently, we introduced in [1] a new class of differentiable structure called pseudo Cauchy Riemann structure on $\bar{M}$ defined by a real tensor field $\bar{J}$ of type $(1,1)$ at every point of $\bar{M}$ satisfying

$$
\bar{J}^{2}=\lambda^{2} I,
$$

where $\lambda$ is a nonzero real function on $\bar{M}$. Suppose $(\bar{M}, \bar{g})$ admits a real $r$-dimensional distribution $D$ where $\bar{g}$ is its metric tensor. Following are three mutually exclusive cases of the causal character (see O'Neill [2]) of $g / D$ : (a) $g / D$ is Riemannian, (b) $g / D$ is semi-Riemannian, and (c) $g / D$ is lightlike. If (a) or (b) holds, then following the terminology of Newlander and Nirenberg [3], a manifold $\bar{M}$ with a PCR-structure $(\bar{J}, \lambda)$ admits a realizable PCR-structure (see Penrose [4]) if $D$ is invariant $(\bar{J} D=D)$ with respect to $\bar{J}$ and it is involutive, that is, $[\bar{X}, \bar{Y}] \in D \forall \bar{X}, \bar{Y} \in D$. Then, $(\bar{M}, \bar{g}, \bar{J}, \lambda)$ is called a PCRmanifold satisfying

$$
T \bar{M}=D \oplus D^{\perp}, D \cap D^{\perp}=\{0\}, \operatorname{dim}\left(D^{\perp}\right)=m-r .
$$

In this paper, we assume that PCR-structure is realizable. We quote following two results for any PCR-manifold.
Theorem 1 (see [1]). A PCR-manifold $(\bar{M}, \bar{J}, \lambda)$ is not necessarily even-dimensional if $\lambda$ is everywhere nonzero real function on $\bar{M}$.

Suppose $\bar{M}$ admits a semi-Riemannian metric $\bar{g}$. Then $(\bar{M}, \bar{g}, \bar{J}, \lambda)$ is also an almost metric PCR-manifold if for a nonzero real function $\mu$,

$$
\bar{g}(\overline{J X}, \overline{J Y})=\mu \bar{g}(\bar{X}, \bar{Y}), \quad \forall \bar{X}, \bar{Y} \in \Gamma(T \bar{M}),
$$

that is, $\bar{g}$ compatible with $\bar{J}$ is conformal or homothetic (in particular, isometric) if $\mu$ is constant (in particular, $\mu=1$ ), respectively.

Proposition 1 (see [1]). Suppose an almost metric PCRmanifold $\bar{M}$ admits a 2 -form $\bar{\Omega}$ defined by (3). Then, $\mu=-\lambda^{2}$.

In support of the above results, we presented two odddimensional examples of almost metric PCR-manifolds and one spacetime model. Also, in same paper [1], we introduced a revised version of a contact manifold, called contact pseudo framed (CPF)-manifold $(M, g, f, \lambda)$ by a real tensor field $f$ of type $(1,1)$ and a real function $\lambda$ such that $f^{3}=\lambda^{2} f$, and $T(M)$ splits into a direct sum of two subbundles, 
namely, $\operatorname{im}(f)$ with a pseudo Cauchy Riemann (PCR)structure and 1-dimensional $\operatorname{ker}(f)$. Contrary to the odddimensional contact manifolds, for the first time in the literature, we presented some examples of a class of evendimensional CPF-manifolds and also shown that the metric of PCR and CPF-manifolds is not severely restricted.

The purpose of this paper is to study CPF-manifolds, with focus on their new even-dimensional class, and discuss the similarities and differences compared with the results of odd-dimensional contact manifolds [5] and physical applications compared with one of our paper on contact spacetime manifolds [6]. We also solve some problems proposed in our previous paper [1], propose new open problems, and suggest further research on this topic.

\section{Contact Pseudo Framed (CPF) Manifolds}

Recall from [1] that an $(m+1)$-dimensional manifold $(M, g, f, \lambda, \eta, \xi)$ is called an almost metric contact pseudo framed (CPF) manifold, where $\eta$ is a 1 -form (called contact form); $\xi$ is a vector field, called characteristic vector field; $g$ is a semi-Riemannian metric; and $f$ is a $(1,1)$ ) tensor field satisfying

$$
\begin{aligned}
f^{3}-\lambda^{2} f & =0, \quad \eta(\xi)=1, \\
g(f X, f Y)) & =\mu\left(g(X, Y)-\varepsilon \sigma^{2} \eta(X) \eta(Y)\right), \\
g(X, \xi) & =\varepsilon \sigma^{2} \eta(X), g(\xi, \xi)=\varepsilon \sigma^{2}, \quad \forall X, Y \in T(M) .
\end{aligned}
$$

where $\varepsilon=+1$ or -1 as $\xi$ is spacelike or timelike and $\lambda, \sigma$, and $\mu$ are real nonzero functions. Also, as per previous section, there exists an $m$-dimensional almost PCR-distribution $D$ given by $\left(\bar{J}=f / D, \bar{J}^{2}=\lambda^{2} I, \eta=0\right)$ which is not necessarily even-dimensional and $D$ is spacelike or timelike or lightlike, where $\xi$ is never a lightlike vector.

Example 1. Let $\left\{\xi_{1}, \xi_{2}, \xi_{3}, \xi_{4}, \xi_{5}, \xi\right\}$ be a basis for $T_{p} M$ at a point $p$ of a 6- dimensional semi-Riemannian manifold $(M, g)$, with a real tensor field $f$ of type $(1,1)$ and two real nonzero functions $\lambda$ and $\mu$. Suppose

$$
\begin{aligned}
f \xi_{1} & =\lambda \xi_{1}, f \xi_{2}=\xi_{3}, f \xi_{3}=\lambda^{2} \xi_{2}, \\
f \xi_{4} & =\xi_{5}, f \xi_{5}=\lambda^{2} \xi_{4}, f \xi=0, \\
g\left(\xi_{a}, \xi_{a}\right) & =\sigma_{a}^{2} \quad \forall a \in(1, \ldots, 5), g(\xi, \xi)=\varepsilon \sigma^{2} .
\end{aligned}
$$

Then, it is easy to see that $f^{2} \xi_{a}=\lambda^{2} \xi_{a} \forall a \in(1, \ldots, 5)$. Therefore, we have a 5-dimensional PCR-distribution $\left(D, \bar{J}=f / D, \bar{J}^{2}=\lambda^{2} I\right)$ generated by $\left\{\xi_{1}, \ldots, \xi_{5}\right\}$ and a complementary 1-dimensional null operator $\widetilde{D}$ such that $T(M)$ splits into a direct sum of two subbundles, namely, $\operatorname{im}(f)$ (with a PCR-structure) and $\operatorname{ker}(f)$. Using the metric compatible equation (3) for the PCR-subspace, we get

$$
\begin{aligned}
& g\left(f \xi_{1}, f \xi_{1}\right)=\mu g\left(\xi_{1}, \xi_{1}\right)=\mu \sigma_{1}^{2}=\lambda^{2} \sigma_{1}^{2} \Rightarrow \mu=\lambda^{2}, \\
& g\left(f \xi_{2}, f \xi_{2}\right)=\lambda^{2} g\left(\xi_{2}, \xi_{2}\right)=\lambda^{2} \sigma_{2}^{2}=g\left(\xi_{3}, \xi_{3}\right)=\sigma_{3}^{2}, \\
& g\left(f \xi_{3}, f \xi_{3}\right)=\lambda^{2} \sigma_{3}^{2}=\lambda^{4} \sigma_{2}^{2} .
\end{aligned}
$$

Cancelling $\lambda^{2}$, we get $\sigma_{3}^{2}=\lambda^{2} \sigma_{2}^{2}$. Similarly, for the vectors $\left(\xi_{4}, \xi_{5}\right)$, we get $\sigma_{5}^{2}=\lambda^{2} \sigma_{4}^{2}$. Here, we used the same symbol $f$ for the PCD-subspace. Using the above data and taking $X=\Sigma_{a} \eta^{a}(X) \xi_{a}+\eta(X) \xi$, we have

$$
\begin{aligned}
g(X, Y) & =\sigma_{1}^{2} \eta^{1}(X) \eta^{1}(Y)+\sigma_{2}^{2}\left(\eta^{2}(X) \eta^{2}(Y)+\lambda^{2} \eta^{3}(X) \eta^{3}(Y)\right)+\sigma_{4}^{2}\left(\eta^{4}(X) \eta^{4}(Y)+\lambda^{2} \eta^{5}(X) \eta^{5}(Y)\right)+\varepsilon \sigma^{2} \eta(X) \eta(Y), \\
f X & =\lambda \eta^{1}(X) \xi_{1}+\eta^{2}(X) \xi_{3}+\lambda^{2} \eta^{3}(X) \xi_{2}+\eta^{4}(X) \xi_{5}+\lambda^{2} \eta^{5}(X) \xi_{4}, \\
f^{2} X & =\lambda^{2}(X-\eta(X) \xi), \quad f^{3} X=\lambda^{2} f X, \quad \operatorname{rank}(f)=5, \\
g(f X, f Y) & =\lambda^{2}\left(g(X, Y)-\varepsilon \sigma^{2} \eta(X) \eta(Y)\right), \quad \forall X, Y \in \Gamma(\mathrm{TM}) .
\end{aligned}
$$

Therefore, $(M, g, f, \lambda, \eta, \xi)$ is an almost metric (Riemannian or Lorentzian as $\varepsilon$ is 1 or -1 , respectively) CPFmanifold.

Remark 1. The above example confirms that contrary to the odd-dimensional almost contact manifolds, there do exist a new class of even-dimensional almost Riemannian or Lorentzian CPF-manifolds. Secondly, if we take the triplet $\left(\varepsilon_{1} \sigma_{1}\right.$, $\left.\varepsilon_{2} \sigma_{2}, \varepsilon_{4} \sigma_{4}\right)$, then it is easy to construct an example of semiRiemannian almost CPF-manifold. So, the metric of this new class is not very restrictive. Finally, we leave it an open problem of taking another set of data for $\left\{f \xi_{1}, \ldots, f \xi_{5}\right\}$ for constructing more general examples of semi-Riemannian even- and odddimensional almost CPF-manifolds. In particular, if $\xi$ is global, then for the Lorentzian case (following the terminology used in [6]), we say that $M$ is a spacetime manifold. As an application, see reference [1] where it has been shown that 4-dimensional (in general 2n-dimensional) de-Sitter and Robertson-Walker spacetimes are physical examples of almost CPF-spacetime manifolds. Moreover, all the physical applications presented in an earlier paper [6] on odd-dimensional contact spacetimes will also hold for even-dimensional almost CPF-spacetimes.

Suppose $M$ admits a 2 -form $\Omega$ defined by

$$
\Omega(X, Y)=g(X, f Y), \quad \forall X, Y \in \Gamma(\mathrm{TM}) .
$$

It is easy to show that for $M$ admitting above 2-form $\Omega$, then $\mu=-\lambda^{2}$. We say that $M$ is almost CPK-manifold (briefly, ACPK-manifold) if $\Omega$ is closed, i.e., $d(\Omega)=0$, and it 
is called metric CPK-manifold if its CPF-structure is normal, i.e., its torsion tensor $T_{f(\lambda)}$ is zero, i.e.,

$$
T_{f(\lambda)} \equiv N_{f(\lambda)}-\lambda^{2} \mathrm{~d} \eta \otimes \xi=0,
$$

where $N_{f(\lambda)}$ is the pseudo Nijenhuis tensor of $f$.

2.1. ACPF-Hypersurfaces. Let $\left(M_{m+1}, f, g, \lambda, \eta, \xi\right)$ be an almost metric CPF-hypersurface of a metric PCR-manifold $\left(\bar{M}_{m+2}, \bar{J}, \bar{g}\right)$. Then, as explained in the previous section, there exists an $m$-dimensional almost PCR-distribution $D$ given by $\left(\bar{J}=f / D, \bar{J}^{2}=\lambda^{2} I, \eta=0\right)$, and $g / D$ is either nondegenerate or degenerate. In this subsection, we assume that $g / D$ is nondegenerate.

Mathematical Model. Let $\bar{M}_{m+2}=M_{m+1} \times R$ be a product manifold where $R$ is a 1 -dimensional affine space and $M_{m+1}$ is an almost metric CPF-manifold with respect to a metric connection $\nabla$ satisfying $\nabla g=0$. Denote a vector field on $\bar{M}_{m+2}$ by $\bar{X}=(X, \Phi \partial / \partial x)$ where $X$ is tangent to $M_{m+1}, x$ is coordinate of $R$, and $\Phi$ is a real function on $\bar{M}_{m+2}$. Define a $(1,1)$ tensor field $\bar{J}$ and a semi-Riemannian metric $\bar{g}$ on $\bar{M}_{m+2}$ by

$$
\begin{aligned}
\bar{J}\left(X, \Phi \frac{\mathrm{d}}{\mathrm{d} x}\right) & =\left(f X-\Phi \xi, \lambda^{2} \eta(X) \frac{\mathrm{d}}{\mathrm{d} x}\right), \\
\bar{g}\left(\left(X, \Phi \frac{\mathrm{d}}{\mathrm{d} x}\right),\left(Y, \theta \frac{\mathrm{d}}{\mathrm{d} x}\right)\right) & =\mu g(X, Y)+\varepsilon \theta \Phi \sigma^{2},
\end{aligned}
$$

where $\theta$ is also a real function on $\bar{M}_{n}$. Using above two equations, it is easy to show that $\bar{J}^{2}=\lambda^{2} I$ and $\bar{g}(\overline{J X}, \overline{J Y})=\mu \bar{g}(\bar{X}, \bar{Y})$. We further assume that $\overline{\nabla J}=0$. Therefore, $\left(\bar{M}_{m+2}, \bar{J}, \bar{g}\right)$ is a metric PCR-manifold with respect to a metric connection $\bar{\nabla}$ satisfying $\bar{\nabla} \bar{g}=0$.

Theorem 2. Let $\left(M_{m+1}, f, g, \lambda, \eta, \xi\right)$ be an almost metric CPF-hypersurface of a metric PCR-manifold $\left(\bar{M}_{m+2}, \bar{J}, \bar{g}\right)$ which satisfies (10) and (11). If $M$ admits a 2-form $\Omega$ and $\lambda$ is constant, then

(a) $\Omega$ is closed, that is, $d(\Omega)=0$
(b) $£_{\xi} g(X, Y)=\varepsilon \sigma^{2}\left(X\left(\sigma^{2}\right) \eta(Y)+Y\left(\sigma^{2}\right) \eta(X)\right)$
(c) $\xi$ is Killing if $\sigma$ is also constant

Proof 1. Using $\mu=-\lambda^{2}$, a straightforward computation of

$$
2 \bar{g}\left(\bar{\nabla}_{(X, 0)}(Y, 0),\left(Z, \frac{\mathrm{d}}{\mathrm{d} x}\right)\right), 2 \bar{g}\left(\bar{\nabla}_{(X, 0)}\left(0, \frac{\mathrm{d}}{\mathrm{d} x}\right),\left(Z, \frac{\mathrm{d}}{\mathrm{d} x}\right)\right) \text {, }
$$

provides

$$
\begin{aligned}
& \bar{\nabla}_{(X, 0)}\left(0, \frac{\mathrm{d}}{\mathrm{d} x}\right)=\left(X \sigma^{2}\right) \frac{\mathrm{d}}{\mathrm{d} x}, \\
& \begin{aligned}
\bar{\nabla}_{(X, 0)}(Y, 0)=\nabla_{X} Y+g(X, Y) \operatorname{grad} \lambda^{2}-\left(X \ln \lambda^{2}\right) Y & -\left(Y \ln \lambda^{2}\right) X . \\
\mathfrak{E}_{\xi} g(X, Y) & =g\left(X\left(\sigma^{2}\right) \xi, Y\right)+g\left(Y\left(\sigma^{2}\right) \xi, X\right) \\
& =X\left(\sigma^{2}\right) \varepsilon \sigma^{2} \eta(Y)+Y\left(\sigma^{2}\right) \varepsilon \sigma^{2} \eta(X) \\
& =\varepsilon \sigma^{2}\left(X\left(\sigma^{2}\right) \eta(Y)+Y\left(\sigma^{2}\right) \eta(X)\right) .
\end{aligned}
\end{aligned}
$$

From the above
asy to show that

(1) $d(\Omega)=d(\ln \lambda) \wedge \Omega$

(2) For $\lambda$ constant, $\nabla_{X} \xi=\left(X \sigma^{2}\right) \xi$

Equation (1) implies $d(\Omega)=0$ if $\lambda$ is constant, (a) holds. Now, again using the above two results and $\overline{\nabla J}=0$ provides

$$
\bar{\nabla}_{(X, 0)} \bar{J}\left(0, \frac{\mathrm{d}}{\mathrm{d} x}\right)=\bar{\nabla}_{(X, 0)}(\xi, 0)-\bar{J}\left(0,\left(X \sigma^{2}\right) \frac{\mathrm{d}}{\mathrm{d} x}\right) .
$$

Then, (2) follows by using (15) and $\lambda$ constant in (14). Therefore,
Consequently, (b) holds and (c) is immediate.

Remark 2. Recall an open problem in [1] to find the condition on pair $(\lambda, \mu)$ for almost Kähler $\longrightarrow$ condition $d(\Omega)=0$, and since $(\lambda, \mu)$ are same for almost CPF-manifolds, in Theorem 2, we have shown that ACPFmanifold $\longrightarrow d(\Omega)=0$ if $\mu=-\lambda$ is constant, which also holds for ACPK-manifold $\longrightarrow d(\Omega)=0$ and CPK-manifold if its CPF-structure is normal. In 1990, Chinea and Gonzales 
[7] studied classification of the almost contact metric manifolds into several classes. We leave it an open problem to investigate similar classification of ACPF, ACPK, and CPK metric manifolds. Blair [5] has proved that a contact metric manifold is $\mathrm{K}$-contact manifold if its characteristic vector field $\xi$ is Killing. On the contrary, we have shown in the above theorem that $\xi$ of a CPK-manifold is Killing only if $\sigma$ is also constant. Later on we will discuss an application of the non-Killing condition (b) of this theorem.

Since Example 1 holds for $\mu=\lambda^{2}$, it does not support the condition $\mu=-\lambda^{2}$ of Theorem 2 with $\Omega$, for which we have following example.

Example 2. Suppose $\left\{\xi_{1}, \xi_{2}, \xi_{3}, \xi_{4}, \xi\right\}$ is a basis for $T_{p} M_{5}$ at a point $p$ of a semi-Riemannian manifold $\left(M_{5}, g\right)$ with a real tensor field $f$ of type $(1,1)$ and two real nonzero functions $\lambda$ and $\mu$ on $M$. Consider, as per Theorem $2,\left(M_{5}, g\right)$ is hypersurface of a metric PCR-manifold $\left(\bar{M}_{6}, \bar{J}, \bar{g}\right)$ which satisfies (13) and (14) with $\lambda$ constant. Let

$$
f \xi_{1}=\xi_{2}, f \xi_{2}=\lambda^{2} \xi_{1}, f \xi_{3}=\xi_{4}, f \xi_{4}=\lambda^{2} \xi_{3}, f \xi=0 .
$$

From the above, we get $f^{2} \xi_{a}=\lambda^{2} \xi_{a}, \forall a \in\left\{\xi_{1}, \ldots, \xi_{4}\right\}$. Therefore, as explained in the previous example, we can use the metric compatible equation (3). Assume that $M$ admits a 2 -form $\Omega$ defined by (8) so $\mu=-\lambda^{2}$.

Take $g\left(\xi_{1}, \xi_{1}\right)=\sigma_{1}^{2}, g\left(\xi_{3}, \xi_{3}\right)=\sigma_{3}^{2}$, and $g(\xi, \xi)=\varepsilon \sigma^{2}$, where $\sigma_{1}, \sigma_{3}$, and $\sigma$ are real functions on $M$. Then, using (3), we get $g\left(f \xi_{1}, f \xi_{1}\right)=-\lambda^{2} g\left(\xi_{1}, \xi_{1}\right)=-\lambda^{2} \sigma_{1}^{2}=g\left(\xi_{2}, \xi_{2}\right)$. Thus, $g\left(\xi_{2}, \xi_{2}\right)=-\lambda^{2} \sigma_{1}^{2}$. Similarly, we get $g\left(\xi_{4}, \xi_{4}\right)=-\lambda^{2} \sigma_{3}^{2}$. With this data and $X=\eta^{1}(X) \xi_{1}+\eta^{2}(X) \xi_{2}+\eta^{3}(X) \xi_{3}+$ $\eta^{4}(X) \xi_{4}+\eta(X) \xi$, we have

$$
\begin{aligned}
g(X, Y) & =\sigma_{1}^{2}\left[\eta^{1}(X) \eta^{1}(Y)-\lambda^{2} \eta^{2}(X) \eta^{2}(Y)\right]+\sigma_{3}^{2}\left[\eta^{3}(X) \eta^{3}(Y)-\lambda^{2} \eta^{4}(X) \eta^{4}(Y)\right]+\varepsilon \sigma^{2} \eta(X) \eta(Y), \\
f X & =\eta^{1}(X) \xi_{2}+\lambda^{2} \eta^{2}(X) \xi_{1}+\eta^{3}(X) \xi_{4}+\lambda^{2} \eta^{4}(X) \xi_{3} \\
f^{2} X & =\lambda^{2}(X-\eta(X) \xi), f^{3} X=\lambda^{2} f X, \operatorname{rank}(f)=4 \\
g(f X, f Y) & =-\lambda^{2}\left(g(X, Y)-\varepsilon \sigma^{2} \eta(X) \eta(Y)\right), \quad \forall X, Y \in \Gamma(\mathrm{TM}) .
\end{aligned}
$$

Thus, in support of Theorem 2, we have an example of an odd-dimensional semi-Riemannian ACPF-hypersurface $\left(M_{5}, f, g, \lambda, \eta, \xi\right)$, of a metric PCR-manifold $\left(\bar{M}_{6}, \bar{g}\right)$, which admits a 2 -form $\Omega$ and spacelike or timelike $\xi$ as $\varepsilon$ is 1 or -1 . The case of even-dimensional semi-Riemannian ACPFhypersurfaces which admit a 2 -form $\Omega$ is left open.

Application 1. Here, we present an application of the conclusion (b) of Theorem 2 for a $(m+1)$-dimensional ACPF-hypersurface $(M, f, g, \lambda, \eta, \xi)$ of a $(m+2)$-dimensional metric PCR-manifold $(\bar{M}, \bar{g}, \lambda)$, which admits a closed 2 -form $\Omega$ if $\lambda$ is constant. This means that

$$
\mathfrak{E g}(X, Y)=\varepsilon \sigma^{2}\left(X\left(\sigma^{2}\right) \eta(Y)+Y\left(\sigma^{2}\right) \eta(X)\right) .
$$

Consider a local coordinates system $\left\{x^{1}, \ldots, x^{m}, x\right\}$ of $T_{p} M$ at a point $p$ of $M$. We know that for the tangent vector $\xi \in T_{p} M$, there is its dual $\eta=T_{p} M \longrightarrow R$. If we set $X\left(\sigma^{2}\right)=$ $d\left(\sigma^{2}\right)(X)$ where $d$ is the ordinary derivative, then relating this with $\eta$, we get $X\left(\sigma^{2}\right)=d\left(\sigma^{2}\right)(X)=\eta(X)$. Putting this value of $X\left(\sigma^{2}\right)$ in (19), we get

$$
\mathfrak{£}_{\xi} g(X, Y)=2 \varepsilon \sigma^{2} \eta(X) \eta(Y)
$$

For an application of the above data, we now prefer using the local coordinates system $\left(x^{i}\right)$. Suppose, in general, the Ricci tensor $R_{i j}$ of a CPF-hypersurface of a semi-Riemannian manifold is of the form as follows:

$$
R_{i j}=a \alpha g_{i j}+b \beta^{2} \eta_{i} \eta_{j}
$$

where $a, b$ are constants and $\alpha, \beta$ are functions on $M$. A particular case of above expression (21) of Ricci tensor was used by Yano (see page 163 in [8]) for a real hypersurface $M$ of a Kaehlerian manifold for which $\alpha=\beta=1$, and he called $M$ pseudo-Einstein and Einstein only if $b=0$. Now, we need following brief information on a symmetry called curvature collineation (CC) defined and studied by Katzin et al. [9] on the existence of a vector field $\xi$ of an $(m+1)$-dimensional semi-Riemannian manifold $(M, g)$ which leaves its curvature tensor invariant. This means

$$
\mathfrak{E}_{\xi} R_{j k l}^{i}=0 \text {. }
$$

Obviously, (22) implies, by contraction, that $\xi$ is also a Ricci collineation (RC) vector field, that is,

$$
\mathfrak{E}_{\xi} R_{i j}=0 \text {. }
$$

However, it is easy to see that RC does not imply CC. In general, we set

$$
\mathfrak{E}_{\xi} g_{i j}=\xi_{i ; j}+\xi_{j ; i}=P_{i j}
$$

The following form of the Lie-derivative of curvature tensor is well-known:

$$
\mathfrak{E}_{\xi} R_{j k m}^{i}=\left(\mathfrak{E}_{\xi} \Gamma_{j m}^{i}\right)_{; k}-\left(\mathfrak{E}_{\xi} \Gamma_{j k}^{i}\right)_{; m}
$$

For a CC vector field $\xi$, using (22)-(25), the following holds:

$$
\begin{aligned}
\mathfrak{E}_{\xi} R_{j}^{i} & =-R_{j}^{k} P_{k}^{i}, \\
\mathfrak{E}_{\xi} r & =r^{\prime} \equiv-2 R_{j}^{i} \xi_{; i}^{j}, \\
\mathfrak{E}_{\xi} C_{j k m}^{i} & =T_{j k m}^{i},
\end{aligned}
$$

where $r$ and $C_{j k m}^{i}$ are the scalar curvature and the conformal curvature tensor, respectively, and 


$$
T_{j k m}^{i} \equiv \frac{1}{(m-1)}\left[P_{j m} R_{k}^{i}-P_{j k} R_{m}^{i}+g_{j k} R_{m}^{e} P_{e}^{i}-g_{j m} R_{k}^{e} P_{e}^{i}\right]+\frac{1}{(m)(m-1)}\left[\delta_{m}^{i}\left(r P_{j k}-r^{\prime} g_{j k}\right)-\delta_{k}^{i}\left(r P_{j m}-r^{\prime} g_{j m}\right)\right]
$$

In particular, it follows from the above third identity that if $M$ is conformally flat, that is, if $C$ vanishes, then $T$ vanishes. However, the converse does not hold, so, in general, following problem remains open.

Condition(s) on $M$ are found with a CC symmetry such that $T$ vanishes.

Equation (25) raises the question of finding possible values for the tensor $P_{i j}$ which represents the change in $g$, with respect to vector field $\xi$. For this purpose, recall that any curvature tensor satisfies following identity: $g_{i j} R_{k m e}^{i}+g_{i k} R_{j m e}^{i}=0$. Taking Lie-derivative of this identity with respect to $\xi$, using (22) and (25), we state the following.

Proposition 2. A necessary condition for a vector field $\xi$ to be a CC vector is that the following curvature identity holds:

$$
P_{i j} R_{k m e}^{i}+P_{i k} R_{j m e}^{i}=0 .
$$

Since the above identity places no restriction on CC vector field $\xi$, it is reasonable to use it for the Ricci curvature $R_{i j}$ of CPF-hypersurface $M$, satisfying some prescribed value of (21). In support of this, we cite following two references of conformally flat $T$ vanishes and nonconformally flat () $M$, respectively:

(1) Set $a=-2, b=2$, and $\beta^{2}=\varepsilon \sigma^{2}$ in general equation (21). Using this and (20) in (21), we get

$$
\mathfrak{E}_{\xi} \mathfrak{g}_{i j}=2 \alpha g_{i j}+R_{i j}
$$

Katzin et al. [9] have proved that the necessary condition for a non-Einstein conformally flat manifold $(M, g, \xi)$ to admit a curvature collineation vector field $\xi$ is

$$
\mathfrak{E}_{\xi} g_{i j}=2 \alpha g_{i j}+\Omega R_{i j}
$$

where $\Omega$ and $\alpha$ are functions on $M$. Thus, for $\Omega=1$, the above equation represents our prescribed relation (29) for a non-Einstein conformally flat CPFhypersurface $(M, f, g, \lambda, \eta, \xi)$ of an almost metric CPF-manifold $(\bar{M}, \bar{g}, \lambda)$ such that $\xi$ is also a CC vector field.

(2) Here, we set $a=0, b=1 / 2$, and $\beta^{2}=\varepsilon \sigma^{2}$ in general equation (21). Using this and (20) in (21), we get

$$
\mathfrak{E}_{\xi} g_{i j}=2 R_{i j}
$$

which is a subcase of CC known as affine collineation (AC) [10], where the Ricci tensor $R_{i j}$ of $M$ is covariant constant $\left(\nabla R_{i j}=0\right)$. Using this symmetry, Grycak [11] has proved that a non-Einstein conformally recurrent manifold (i.e., $\nabla C=\mathrm{d} \theta \otimes C$, where $C$ is the conformal curvature tensor and $\mathrm{d} \theta$ is an exact recurrent form) which is neither conformally flat nor recurrent admits an AC vector field $\xi$ satisfying (31) for a non-Einstein conformally recurrent CPF-hypersurface $(M, f, g, \lambda, \eta, \xi)$ of an almost metric CPF-manifold $(\bar{M}, \bar{g}, \lambda)$. On conformally recurrent manifolds, we refer [12].

Application 2. Here, we use the following conformal collineation symmetry introduced by Tashiro [13]:

Definition 1. An $(m+1)$ dimensional semi-Riemannian manifold $(M, g)$ admits a conformal collineation symmetry defined by a vector field $\xi$ if

$$
\mathfrak{E}_{\xi} \Gamma_{i j}^{k}=\delta_{i}^{k} \alpha_{j}+\delta_{j}^{k} \alpha_{i}-g_{i j} \alpha^{k},
$$

where $\Gamma_{i j}^{k}$ denotes Christoffel's symbols, $\alpha$ is a function and $\alpha_{j}=\partial_{j}(\alpha)$, and $\xi$ is called an Affine Conformal Vector (ACV) field of $M$.

Proposition 3 (see [10]). A vector field $\xi$ on a semi-Riemannian manifold $(M, g)$ is an $A C V$ if and only if

$$
\mathfrak{E}_{\xi} g=2 \alpha g+K
$$

where $K$ is a $(0,2)$ covariant constant $(\nabla K=0)$ symmetric tensor.

An ACV reduces to a conformal Killing vector, briefly denoted by $\mathrm{CKV}$, if $\mathrm{K}$ is proportional to $g$. Thus, an ACV deviates from a CKV field if there exists a second-order covariant constant symmetric tensor $K \neq g$. On the existence of an ACV and specific restrictions on the ambient manifold $M$, we recall that, in 1932, Eisenhart [14] proved "If a Riemannian manifold $M$ admits such a tensor $K$, independent of $g$, then $M$ is reducible." This means that $M$ is a product manifold of the form $\left(\bar{M}=M_{1} \times M_{2}, \bar{g}=g_{1} \oplus g_{2}\right)$. In 1926, H. Levy [15] proved that "A second-order covariant constant nonsingular symmetric tensor in a space of constant curvature is proportional to the metric tensor." Thus, a semi-Riemannian manifold of constant curvature admits no ACV other than a $\mathrm{CKV}$. So, $M$ with proper ACV is restricted to manifolds with nonconstant curvature. Physically, for example, Minkowski, de-Sitter, or anti-de-Sitter spacetimes do not admit an ACV. In particular, an ACV is called an affine vector, briefly denoted by $\mathrm{AV}$, if $\alpha=0$. For basic details on ACV, see Tashiro [13], Paterson [16], Duggal [10], Mason-Martens [17], and others referred therein. For detailed information on curvature, affine, and conformal collineations, we refer [18]. Following is a reference having a link of CPF-hypersurfaces with conformal collineation manifolds.

(3) We know that $(M, g)$ is Ricci symmetric if $\nabla R_{i j}=0$. Levine-Katzin [19] have proved that if a conformally flat 
manifold $(M, g)$ admits a conformal collineation symmetry, with CKV vector field $\xi$, then $K_{i j}=a g_{i j}+b R_{i j}$ for some constants $a$ and $b$ and $M$ is Ricci symmetric. Thus, for $a=0$ and $b=1$, the ACV vector field $\xi$ satisfies the prescribed relation (33) for a Ricci symmetric CPF-hypersurface $(M, f, g, \lambda, \eta, \xi)$ of an almost metric CPF-manifold $(\bar{M}, \bar{g})$. Based on the above three references, we state following Corollary of Theorem 2:

Corollary 1. Let $\left(M_{m+1}, f, g, \lambda, \eta, \xi\right)$ be an almost metric CPF-hypersurface of a metric PCR-manifold $\left(\bar{M}_{m+2}, \bar{J}, \bar{g}\right)$, with constant $\lambda$ and $M$ admits 2 -form $\Omega$. Then, $(M, g)$ admits curvature or affine or conformal collineations symmetries for prescribed values of the general equation (21).

The picture views of this link with three curvature symmetries are as follows:

$$
\mathrm{CPFH} \stackrel{\mathrm{CC}}{\longrightarrow} \mathrm{CFM},
$$

where CPFH and HFM denote CPF-hypersurface and conformally flat manifold, respectively, and

$$
\mathrm{CPFH} \stackrel{\mathrm{AC}}{\longrightarrow} \mathrm{CRM},
$$

where CRM denotes conformally recurrent manifold.

$$
\mathrm{CPFH} \stackrel{\mathrm{ACV}}{\longrightarrow} \mathrm{RCRM},
$$

where RCRM denotes reducible conformally recurrent manifold.

$$
\mathfrak{E}_{\xi} \bar{g}_{i j}=2 \alpha g_{i j}-2 R_{i j}
$$

Application 3. Here, we need the following brief on the concept of Ricci flow for Riemannian manifolds introduced by Hamilton [20] in 1982. Let $(M, g)$ be an $(m+1)$-dimensional Riemannian manifold. The Ricci flow on $M$ is defined by the following evolving equation:

where $\xi$ is a vector field on $M$ and $\alpha$ is a constant. The vector field $\xi$, satisfying equation (37), is called Ricci soliton vector and $(M, g, \alpha, \xi)$ is called Ricci soliton (briefly RS) manifold which is said to be shrinking, steady, or expanding as $\alpha$ is positive, zero, or negative, respectively. The Ricci soliton manifolds are natural extension of Einstein manifolds and are selfsimilar (homothetic) solutions, called Ricci solitons. Basic details and a collection of research papers on this area for the Riemannian case are available in $[21,22]$, respectively. There are few papers on Ricci solitons for semi-Riemannian (in particular, Lorentzian) manifolds (for example, see Crasmareanu [23], Brozos-Vazquez et al. [24], and Onda [25]). In year 2011, Pigola et al. [26] introduced a modified concept of the Ricci solitons equation called almost Ricci solitons (briefly, ARS) by allowing the soliton constant $\alpha$ to be a variable function. So far, we know following references on ARS-manifolds: Barros et al.
[27], Barros-Riberiro [28], Sharma [29], Wang [30], and Duggal [31]. Following is a link between the CC symmetry of CPF-hypersurfaces and ARS and RS evolving equation (37) as $\alpha$ is a variable function or a constant.

(4) Grycak [11] has proved that a non-Einstein conformally recurrent manifold (i.e., $\nabla C=\mathrm{d} \theta \otimes C$, where $C$ is the conformal curvature tensor and $\mathrm{d} \theta$ is an exact recurrent form) which is neither conformally flat nor recurrent admits a vector field $\xi$ satisfying (30) such that

$$
\mathfrak{E}_{\xi} \mathcal{g}_{i j}=2 \alpha g_{i j}+\Omega R_{i j}, \quad \Omega=\text { nonzero function on } M \text {. }
$$

And $\alpha$ is a constant. This relates with the Ricci soliton equation (37) if we take $\Omega=-2$ so $(M, g, \alpha, \xi)$ is a RSmanifolds. Although Grycak did not discuss a link of his result with a symmetry vector, but the above relation is a particular case of the general equation (21), it is reasonable to assume that this reference has a link with CC or AC symmetries. Based on the above, we state following Corollary of Theorem 2:

Corollary 2. Let $\left(M_{m+1}, f, g, \lambda, \eta, \xi\right)$ be an almost metric CPF-hypersurface of a metric PCR-manifold $\left(\bar{M}_{m+2}, \bar{J}, \bar{g}\right)$, with constant $\lambda$ and $M$ admits 2-form $\Omega$. Then, $(M, g)$ is also almost Ricci soliton or Ricci soliton for some prescribed values of the general equation (21) as $\alpha$ is a variable function or a constant.

The picture views of this link with the almost Ricci soliton and Ricci soliton manifolds are as follows:

$$
\mathrm{CPFH} \stackrel{\mathrm{CC}}{\longrightarrow} \mathrm{ARSM} \stackrel{\mathrm{CC}}{\longrightarrow} \mathrm{RSM},
$$

where $\mathrm{CPFH}$, ARSM, and RSM denote CPF-hypersurface, almost Ricci soliton, and Ricci soliton manifolds, respectively.

Remark 3. Although we have some references with specific prescriptions for the unknown tensor $P$ linking the CPF-hypersurfaces with three types of curvature symmetries and almost Ricci soliton manifold, it is reasonable to assume that such a link may also hold for some other types of CPF-hypersurfaces of semi-Riemannian manifolds. Therefore, we leave it an open problem to research on deeper study of possible solutions of equation (21) linking the CPF-manifolds with a variety of other types of semi-Riemannian manifolds. Presently, three curvature symmetry manifolds and almost Ricci soliton manifolds are among most important topics of research in differential geometry so their link established with semi-Riemannian CPF-hypersurfaces is expected to produce substantial useful original results on geometry and physics of this new class of ACPF-manifolds with applications. 


\section{Lightlike ACPF-Manifolds}

For this section, we need the following brief information on lightlike manifolds taken from the study by Duggal-Sahin [32] (pages 30-40). Let $\left(M_{m+1}, f, g, \lambda, \eta, \xi\right)$ be an almost metric CPF-manifold which admits a 2 -form $\Omega$. Suppose its PCR-distribution $D(\bar{J}=f / D)$ is lightlike having a degenerate induced metric $g / D$. Let $(N, g / D)$ be the lightlike PCRsubspace of $M$ with respect to its distribution $D$. This means that there exists a vector field say $V \neq 0$ in $N$ such that $g(V, X)=0, \forall X \in \mathrm{TN}$. Let $\operatorname{Rad} \mathrm{TN}$ be the radical distribution of $D$, with respect to $g / D$. Then,

$$
\begin{aligned}
& \operatorname{Rad} \mathrm{TN}\{V \in \mathrm{TN}) ; g / N(V, X)=0, \forall X \in \mathrm{TN}\}, \\
& \mathrm{TN}=\operatorname{Rad} \mathrm{TN} \oplus S(\mathrm{TN}), \quad \operatorname{Rad} \mathrm{TN} \cap S(\mathrm{TN})=\{0\}, \\
& \mathrm{TM}=\operatorname{Rad} \mathrm{TN} \oplus S(\mathrm{TN}) \oplus\{\xi\},
\end{aligned}
$$

where $S(\mathrm{TN})$ is nondegenerate complementary (but not orthogonal) screen distribution of $\operatorname{RadTN}$ in TN. Let $\operatorname{dim}(\operatorname{Rad} \mathrm{TN})=r \geq 1$. Then, $\operatorname{dim}(S(\mathrm{TN}))=m-r \quad$ and $\operatorname{dim}(N)=m$. In this way, $\left(M_{m+1}, f, g, \lambda, \eta, \xi\right)$ is called an $(m+1)$-dimensional $r$-lightlike ACPF-manifold. In this section, we show that there do exist even- and odd-dimensional lightlike ACPF-manifolds with degenerate induced metric which admit a 2 -form $\Omega$. Following is an evendimensional example.
Example 3. Suppose $\left\{\xi_{1}, \ldots, \xi_{2 n-1}, \xi\right\}$ is a basis for $T_{p} M$ at a point $p$ of a $2 n$-dimensional $(n>1)$ metric manifold $(M, g)$ with a real tensor field $f$ of type $(1,1)$ and two real nonzero functions $\lambda$ and $\mu$ on $M$. Let

$$
f \xi_{1}=\lambda \xi_{1}, f \xi_{a}=\xi_{a+1}, f \xi_{a+1}=\lambda^{2} \xi_{a}, \quad \forall a \in(2, \ldots, 2 n-1), f \xi=0 .
$$

Then, it is easy to see that $f^{2} \xi_{a}=\lambda^{2} \xi_{a}, \forall a \epsilon$ $(1, \ldots, 2 n-1)$. Therefore, we have a $(2 n-1)$-dimensional PCR-distribution $\left(D, \bar{J}=f / D, \bar{J}^{2}=\lambda^{2} I\right)$ generated by $\left\{\xi_{1}, \ldots, \xi_{2 n-1}\right\}$ and a complementary 1-dimensional null operator $\widetilde{D}$ such that $T(M)$ splits into a direct sum of two subbundles, namely, $\operatorname{im}(f)$ (with a PCR-structure) and $\operatorname{ker}(f)$. Let $N$ be the PCR-subspace of $M$, with respect to its distribution D. Therefore, we can use the metric compatible equation (3) for the PCR-subspace $(N, g / D)$ of $(M, g)$. Assume $M$ admits a 2 -form $\Omega$ defined by (8) so that $\mu=-\lambda^{2}$. From $f \xi_{1}=\lambda \xi_{1}$, we get $g\left(f \xi_{1}, f \xi_{1}\right)=-\lambda^{2} g\left(\xi_{1}, \xi_{1}\right)=\lambda^{2} g\left(\xi_{1}, \xi_{1}\right)$. This implies that $g\left(\xi_{1}, \xi_{1}\right)=0$ so $\xi_{1}$ is a null vector. Let $g\left(\xi_{a}, \xi_{a}\right)=\sigma_{a}^{2}$ and $g(\xi, \xi)=\varepsilon \sigma^{2}$ for each $a \in(2, \ldots, 2 n-1)$, where each $\sigma_{a}$ and $\sigma$ are real nonzero functions on $M$. Then, $g\left(f \xi_{a}, f \xi_{a}\right)=-\lambda^{2} g\left(\xi_{a}, \xi_{a}\right)=g\left(\xi_{a+1}, \xi_{a+1}\right)$ implies that $\sigma_{a+1}^{2}=-\lambda^{2} \sigma_{a}, \forall a \in(2, \ldots, 2 n-1)$. Using this data and $X=\Sigma_{a} \eta^{a}(X) \xi_{a}+\eta(X) \xi, \forall a \in(1, \ldots, 2 n-1)$, we have

$$
\begin{aligned}
g(X, Y)= & \sigma_{2}^{2}\left(\eta^{2}(X) \eta^{2}(Y)-\lambda^{2} \eta^{3}(X) \eta^{3}(Y)\right)+\sigma_{4}^{2}\left(\eta^{4}(X) \eta^{4}(Y)-\lambda^{2} \eta^{5}(X) \eta^{5}(Y)\right) \\
& +\cdots+\sigma_{2(n-1)}^{2}\left(\eta^{2(n-1)}(X) \eta^{2(n-1)}(Y)-\lambda^{2} \eta^{2 n-1}(X) \eta^{2 n-1}(Y)\right)+\varepsilon \sigma^{2} \eta(X) \eta(Y), \\
f X= & \lambda \eta^{1}(X) \xi_{1}+\eta^{2}(X) \xi_{3}+\lambda^{2} \eta^{3}(X) \xi_{2}+\cdots+\left(\eta^{2(n-1)}(X) \xi_{2(n-1)}-\lambda^{2} \eta^{2 n-1}(X) \xi_{2 n-1}\right) \\
f^{3} X= & \lambda^{2} f X, \quad \forall X, Y \in \mathrm{TM} .
\end{aligned}
$$

Thus, $(M, g, f, \lambda, \xi, \mu)$ is a 2 n-dimensional 1-lightlike ACPF-manifold with 1-dimensional radical distribution $\operatorname{Rad} \mathrm{TN}=\left\{\xi_{1}\right\}$ and $2(n-1)$-dimensional screen distribution $S(\mathrm{TN})=\left\{\xi_{2}, \ldots, \xi_{2 n-1}\right\}$ such that

$$
\begin{aligned}
\operatorname{Rad} \mathrm{TN} & \left.=\left\{\xi_{1} \in \mathrm{TN}\right) ; g / N\left(\xi_{1}, X\right)=0, \forall X \in \mathrm{TN}\right\}, \\
\mathrm{TN} & =\operatorname{Rad} \mathrm{TN} \oplus S(\mathrm{TN}), \quad \operatorname{Rad} \mathrm{TN} \cap S(\mathrm{TN})=\{0\}, \\
\mathrm{TM} & =\mathrm{TN} \oplus\{\xi\}=\operatorname{Rad} \mathrm{TN} \oplus S(\mathrm{TN}) \oplus\{\xi\},
\end{aligned}
$$

where $S(\mathrm{TN})$ is nondegenerate complementary (but not orthogonal) screen distribution of Rad TN in TN. $M$ also admits a 2 -form $\Omega$.

Following is an example of odd-dimensional 2-lightlike ACPF-manifold.

Example 4. Suppose $\left\{\xi_{1}, \xi_{2}, \ldots, \xi_{2 n}, \xi\right\}$ is a basis for $T_{p} M$ of a $(2 n+1)$-dimensional $(n>1)$ metric manifold $(M, g)$ with a real tensor field $f$ of type $(1,1)$ and two real nonzero functions $\lambda$ and $\mu$ on $M$. Let

$$
f \xi_{1}=\lambda \xi_{1}, f \xi_{2}=\lambda, f \xi_{a}=\xi_{a+1}, f \xi_{a+1}=\lambda^{2} \xi_{a}, \quad \forall a \in(3, \ldots, 2 n), f \xi=0
$$

Then, it is easy to see that $f^{2} \xi_{a}=\lambda^{2} \xi_{a}, \forall a \in(1, \ldots, 2 n)$. Therefore, we have a $2 n$-dimensional PCR-distribution $\left(D, \bar{J}=f / D, \bar{J}^{2}=\lambda^{2} I\right)$ generated by $\left\{\xi_{1}, \ldots, \xi_{2 n}\right\}$ and a complementary 1-dimensional null operator $\widetilde{D}$ such that $T(M)$ splits into a direct sum of two subbundles, namely, $\operatorname{im}(f)$ (with a PCR-structure) and $\operatorname{ker}(f)$. Let $(N, g / D)$ be the PCR-subspace of $(M, g)$, with respect to its distribution D. Therefore, we use the metric compatible equation (3) for the PCR-subspace $(N, g / D)$ of $(M, g)$ and $M$ admits a 2form $\Omega$ defined by (8) so that $\mu=-\lambda^{2}$. From $f \xi_{1}=\lambda \xi_{1}$, we get $g\left(f \xi_{1}, f \xi_{1}\right)=-\lambda^{2} g\left(\xi_{1}, \xi_{1}\right)=\lambda^{2} g\left(\xi_{1}, \xi_{1}\right)$. This implies that $g\left(\xi_{1}, \xi_{1}\right)=0$ so $\xi_{1}$ is a null vector. Similarly, $g\left(\xi_{2}, \xi_{2}\right)=$ 
0 so $\xi_{2}$ is also a null vector. Let $g\left(\xi_{a}, \xi_{a}\right)=\sigma_{a}^{2}$ and $g(\xi, \xi)=$ $\varepsilon \sigma^{2}$ for each $a \in(3, \ldots, 2 n)$, where each $\sigma_{a}$ and $\sigma$ are real nonzero functions on $M$. Then, $g\left(f \xi_{a}, f \xi_{a}\right)=-\lambda^{2}$ $g\left(\xi_{a}, \xi_{a}\right)=g\left(\xi_{a+1}, \xi_{a+1}\right) \quad$ implies $\quad \sigma_{a+1}^{2}=-\lambda^{2} \sigma_{a}$, $\forall a \in(3, \ldots, 2 n)$. Using this and $X=\Sigma_{a} \eta^{a}(X) \xi_{a}+\eta(X)$ $\xi, \forall a \in(1, \ldots, 2 n)$, we have

$$
\begin{aligned}
g(X, Y)= & \sigma_{3}^{2}\left(\eta^{3}(X) \eta^{3}(Y)-\lambda^{2} \eta^{4}(X) \eta^{4}(Y)\right)+\sigma_{5}^{2}\left(\eta^{5}(X) \eta^{5}(Y)-\lambda^{2} \eta^{6}(X) \eta^{6}(Y)\right) \\
& +\cdots+\sigma_{2 n}^{2}\left(\eta^{2 n-1}(X) \eta^{2 n-1}(Y)-\lambda^{2} \eta^{2 n}(X) \eta^{2 n}(Y)\right)+\varepsilon \sigma^{2} \eta(X) \eta(Y), \\
f X= & \lambda \eta^{1}(X) \xi_{1}+\lambda \eta^{2}(X) \xi_{2}+\eta^{3}(X) \xi_{4}+\lambda^{2} \eta^{4}(X) \xi_{3}+\cdots+\left(\eta^{2 n-1}(X) \xi_{2 n-1}-\lambda^{2} \eta^{2 n}(X) \xi_{2 n}\right) \\
f^{3} X= & \lambda^{2} f X, \quad \forall X, Y \in \mathrm{TM} .
\end{aligned}
$$

Thus, $(M, g, f, \lambda)$ is a $(2 n+1)$-dimensional 2-lightlike ACPF-manifold which admits a 2-dimensional radical distribution $\operatorname{RadTN}=\left\{\xi_{1}, \xi_{2}\right\}$ and $(2 n-2)$-dimensional screen distribution $S(\mathrm{TN})=\left\{\xi_{3}, \ldots, \xi_{2 n}\right\}$ such that

$$
\begin{aligned}
\operatorname{Rad} \mathrm{TN} & \left.=\left\{\xi_{1} \in \mathrm{TN}\right) ; g / N\left(\xi_{1}, X\right)=0, \forall X \in \mathrm{TN}\right\}, \\
\mathrm{TN} & =\operatorname{Rad} \mathrm{TN} \oplus S(\mathrm{TN}), \quad \operatorname{Rad} \mathrm{TN} \cap S(\mathrm{TN})=\{0\}, \\
\mathrm{TM} & =\mathrm{TN} \oplus\{\xi\}=\operatorname{Rad} \mathrm{TN} \oplus S(\mathrm{TN}) \oplus\{\xi\},
\end{aligned}
$$

where $S(\mathrm{TN})$ is nondegenerate complementary (but not orthogonal) screen distribution of Rad TN in TN. $M$ also admits a 2-form $\Omega$.

Finally, we present a physical model of 1-lightlike ACPFhypersurfaces of a metric PCR-globally hyperbolic spacetime. A product manifold $\left(\bar{M}=\mathbf{R} \times N, \bar{g}=-\mathrm{d} t^{2} \oplus G\right)$ is called a globally hyperbolic spacetime manifold if $(N, G)$ is a compact Riemannian manifold and $\bar{M}$ is time oriented by a timelike global vector field $V$. Simple examples are Minkowski, de-Sitter, and Einstein static spacetimes. We refer [33] for details on physical importance of globally hyperbolic spacetimes.

Physical Model. Let $(\bar{M}, \bar{J}, \bar{g}, \lambda)$ be an $(m+2)$-dimensional globally hyperbolic PCR-spacetime with its metric $\bar{g}$ given by

$$
\mathrm{d} s^{2}=-\mathrm{d} t^{2}+\mathrm{d} x^{2}+\bar{g}_{a b} \mathrm{~d} x^{a} \mathrm{~d} x^{b},(a, b=1, \ldots, m)
$$

with respect to a coordinate system $\left(t, x, x^{1}, \ldots, x^{m}\right)$ on $\bar{M}$. Take two null coordinates $u$ and $v$ such that $u=t+x$ and $v=t-x$. Thus, the above metric transforms into a nonsingular metric: $\mathrm{d} s^{2}=-\mathrm{d} u \mathrm{~d} v+\bar{g}_{a b} \mathrm{~d} x^{a} \mathrm{~d} x^{b}$. The absence of $\mathrm{d} u^{2}$ and $\mathrm{d} v^{2}$ in this metric implies that $\{v=$ constant. $\}$ and $\{u=$ constant. $\}$ are lightlike hypersurfaces of $\bar{M}$. Let $(M, g, r=1, v=$ constant.) be one of this lightlike pair with 1-dimensional distribution $\operatorname{Rad}(M)$ generated by the null vector $\left\{\partial_{v}\right\}$ in $M$ and $m$-dimensional Riemannian screen distribution $S(\mathrm{TM})$ with metric $\mathrm{d} \Omega^{2}=g_{a b} \mathrm{~d} x^{a} \mathrm{~d} x^{b}$. In particular, there will be many global timelike vector fields in globally hyperbolic spacetimes $\bar{M}$. If we take a fixed global time function, then its gradient is a global timelike vector field in a given $\bar{M}$. With this choice of a global timelike vector field, we conclude that corresponding lightlike hypersurface admits a global null vector field. Using the Hopf-Rinow theorem, one may choose a screen distribution whose leaf is a complete Riemannian hypersurface of $M$. Thus, we have a physical model of a global 1-lightlike hypersurface of a globally hyperbolic PCR-spacetime manifold. Then, proceeding exactly as in Example 3, it is straight forward to show that $(M, g, r=1, v=$ constant. $)$ is a global 1-lightlike APCF-hypersurface of a globally hyperbolic PCRspacetime manifold $(\bar{M}, \bar{J}, \bar{g}, \lambda)$.

Remark 4. Examples 3 and 4 show that there exist even- and odd-dimensional $r$-lightlike ACPF-manifolds which admit a 2-form $\Omega$. Also, Examples 2-4 confirm that there do exist a new class of odd-dimensional semi-Riemannian and evenor odd-dimensional r-lightlike ACPF-manifolds, in particular, ACPF-hypersurfaces of a metric PCR-manifold $\left(\bar{M}_{m+2}, \bar{J}, \bar{g}\right)$ which admit 2-form $\Omega$. However, we still do not have any example of even-dimensional semi-Riemannian ACPF-manifold with 2-form $\Omega$. We leave this as open problem and propose following for further consideration:

(1) The results presented on ACPF-hypersurfaces in this paper can be extended for general theory of ACPFsubmanifolds of a variety of metric PCR-manifolds by revising the definition of Riemannian contact CRsubmanifolds [8] and contact CR-lightlike submanifolds (see chapter 7 in [32]).

(2) Considerable work has been done on submanifolds of normal contact (also called Sasakian) manifolds (see $[8,34])$. We propose an extension of their results for submanifolds of normal CPF-manifolds.

\section{Data Availability}

The data used to support the findings of this study are available from the corresponding author upon request.

\section{Conflicts of Interest}

The author declares that there are no conflicts of interest regarding the publication of this article.

\section{References}

[1] K. L. Duggal, "Pseudo Cauchy Riemann and framed manifolds with physical applications," International Electronic Journal of geometry, vol. 13, no. 1, pp. 1-14, 2020.

[2] B. O'Neill, Semi-Riemannian Geometry with Applications to Relativity, Academic Press, New York, NY, USA, 1983. 
[3] A. Newlander and L. Nirenberg, "Complex analytic coordinates in almost complex manifolds," Annals of Mathematics, vol. 65, no. 3, pp. 391-404, 1957.

[4] R. Penrose, "Physical space-time and nonrealizable CRstructures," Proceedings of Symposia in Pure Mathematics, vol. 39, pp. 401-422, 1983.

[5] D. E. Blair, "Contact manifolds in Riemannian geometry," in Lecture Notes in MathematicsSpringer-Verlag, Berlin, Germany, 1976.

[6] K. L. Duggal, "Spacetime manifolds and contact structures," International Journal of Mathematics and Mathematical Sciences, vol. 13, pp. 545-554, 1990.

[7] D. Chinea and C. Gonzalez, "A classification of almost contact metric manifolds," Annali di Matematica Pura ed Applicata, vol. 156, no. 1, pp. 15-36, 1990.

[8] K. Yano and M. Kon, CR-submanifolds of Kählerian and Sasakian Manifolds, Birkhauser, Basel, Switzerland, 1983.

[9] G. H. Katzin, J. Levine, and W. R. Davis, "Curvature collineations in conformally flat spaces, I," Tensor N.S.vol. 21, pp. 51-61, 1970.

[10] K. L. Duggal, "Affine conformal vector fields in semi-Riemannian manifolds," Acta Applicandae Mathematica, vol. 23, pp. 275-294, 1991.

[11] W. Grycak, "On affine collineations in conformally recurrent manifolds," Tensor N. S.vol. 35, pp. 45-50, 1981.

[12] T. Adati and T. Miyazawa, "On Riemannian space with recurrent conformal curvature," Tensor (N. S.), vol. 18, pp. 348-354, 1967.

[13] Y. Tashiro, "On conformal collineations," Mathematical Journal of Okayama University, vol. 10, pp. 75-85, 1960.

[14] L. P. Eisenhart, "Symmetric tensors of the second order whose first covariant derivatives are zero," Transactions of the American Mathematical Society, vol. 25, no. 2, p. 297, 1923.

[15] H. Levy, "Symmetric tensors of the second order whose covariant derivatives vanish," Annals of Mathematics, vol. 27, pp. 91-98, 1926.

[16] E. M. Patterson, "On symmetric recurrent tensors of the second order," Quarterly Journal of Mathematics, vol. 2, pp. 151-158, 1951.

[17] D. P. Mason and R. Maartens, "Kinematics and dynamics of conformal collineations in relativity," Journal of Mathematics and Physics, vol. 28, no. 9, pp. 2182-2186, 1985.

[18] K. L. Duggal and R. Sharma, "Symmetries of spacetimes and riemannian manifolds," Kluwer Academic Publishers, New York, NY, USA, 1999.

[19] J. Levine and G. H. Katzin, "Conformally flat spaces admitting special quadratic first integrals, 1. Symmetric spaces," Tensor N. S.vol. 19, pp. 317-328, 1968.

[20] R. Hamilton, "Three-manifolds with positive Ricci curvature," Journal of Differential Geometry, vol. 17, pp. 155-306, 1982.

[21] H. D. Cao, B. Chow, S. C. Chu, and S. T. Yau, Collected papers on Ricci Flow, Series in Geometry and Topology, p. 539, International Press, Vienna, Austria, 2003.

[22] B. Chow and D. Knopf, "The Ricci flow: an introduction," American Mathematical Society, vol. 110, p. 325, 2004.

[23] M. Crasmareanu, "Liouville and geodesic Ricci solitons," Comptes Rendus Mathematique, vol. 347, no. 21-22, pp. 1305-1308, 2009.

[24] M. Brozos-Vázquez, E. García-Río, and G. Fernández, "Locally conformally flat Lorentzian gradient Ricci solitons," Journal of Geometric Analysis, vol. 23, pp. 1196-1212, 201.

[25] K. Onda, "Lorentz Ricci solitons on 3-dimensional Lie groups," Geometriae Dedicata, vol. 147, no. 1, pp. 313-322, 2010.
[26] S. Pigola, M. Rigoli, M. Rimoldi, and A. Setti, "Ricci almost solitons," Annali della Scuola Normale Superiore. Classe di Scienze, vol. 5, pp. 757-799, 2011.

[27] A. Barros, R. Batista, and E. Ribeiro Jr., "Compact almost Ricci solitons with constant scalar curvature are gradient," Monatshefte für Mathematik, vol. 174, no. 1, pp. 29-39, 2014.

[28] A. Barros and E. Riberiro Jr., "Some characterizations for compact almost Ricci solitons," Proceedings of the American Mathematical Society, vol. 140, pp. 1-33, 2012.

[29] R. Sharma, "Almost Ricci solitons and K-contact geometry," Montash Math, vol. 175, pp. 621-628, 2014.

[30] Y. Wang, "Gradient Ricci almost solitons on two classes of almost Kenmotsu manifolds," Journal of the Korean Mathematical Society, vol. 53, no. 5, pp. 1101-1114, 2016.

[31] K. L. Duggal, "A new class of almost Ricci solitons and their physical interpretation," International Scholarly Research Notes, vol. 2016, Article ID 4903520, 6 pages, 2016.

[32] K. L. Duggal and B. Sahin, "Differential geometry of lightlike submanifolds," Frontiers in Mathematics, vol. 476, 2010.

[33] J. K. Beem and P. E. Ehrlich, Global Lorentzian Geometry, Marcel Dekker, Inc., New York, NY, USA, 1981.

[34] A. Bejancu, Geometry of CR-Submanifolds, D. Reidel, Dordrecht, Netherlands, 1986. 